\title{
There and back again: An immunotherapy tale
}

Yizhan Guo, MD, and Alexander Sasha Krupnick, MD

From the Division of Thoracic Surgery, Department of Surgery, University of Virginia, Charlottesville, Va. Received for publication Nov 1, 2017; accepted for publication Nov 12, 2017; available ahead of print Feb 4, 2018.

Address for reprints: Alexander Sasha Krupnick, MD, Department of Surgery and The Carter Immunology Center, University of Virginia, PO Box 800679, Charlottesville, VA 22908 (E-mail: sashak@ virginia.edu).

J Thorac Cardiovasc Surg 2018;155:1771-4 $0022-5223 / \$ 36.00$

Copyright (C) 2017 by The American Association for Thoracic Surgery https://doi.org/10.1016/j.jtcvs.2017.11.102

Feature Editor's Note-We are right now in the center of a revolution in the treatment of patients with advanced cancers using immune checkpoint inhibitors. This is highlighted by the nearly 1000 currently ongoing clinical trials evaluating agents targeting the programmed cell death protein-1 (PD-1) immune checkpoint or its ligand, programed death ligand-1 (PD-L1). ${ }^{1}$ These "immune checkpoints" are regulatory molecules expressed on the surface of $T$ cells that normally function to restrain T-cell overactivation but that are exploited by cancer cells and tumor-associated stromal cells expressing PD-L1 to evade antitumor immune responses. Blocking these inhibitory signals with checkpoint inhibitors "takes the brakes off" the immune system. Such therapy thus leads to an antitumor T-cell immune response. Whereas non-small cell lung cancer (NSCLC) traditionally has been considered a "non-immunogenic" tumor, it is now without question that immune checkpoint inhibitors improve progression-free and overall survival in select patients with advanced and unresectable NSCLC. The quality of responses to PD-1 inhibition in NSCLC range from none to complete and showcase our understanding of NSCLC as a heterogenous disease. To the current variety of histologic (and subhistologic) classes of NSCLC tumors, and of molecular subsets of tumors with targetable mutations, we can add immunologically "hot" and "cold" NSCLCs. Several clinical trials have now demonstrated that the $23 \%$ to $30 \%$ of patients with advanced NSCLC whose tumors express high levels of PD-L1 ( $\geq 50 \%$ tumor cell membranous staining by immunohistochemistry) are likely to have a clinical response to monoclonal antibodies against PD-1. Further, we have learned that responses to PD-1 inhibition can be variable, that the effect on survival is only $\sim 3$ to 4 months of life extension, and that responses are occasionally seen in patients who are PD-L1 negative.

The field is young. In the accompanying Feature Editorial, Guo and Krupnick summarize the results of 3 recent and influential randomized trials of checkpoint inhibitors in lung cancer, KEYNOTE-24, CheckMate 026, and PACIFIC, in the context of the evolving field of tumor

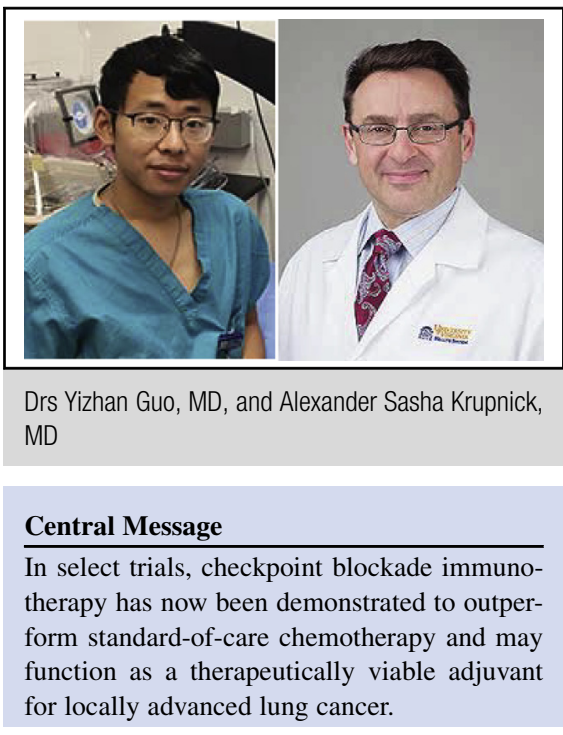

See Editorial Commentary page 1775.

immunotherapy. The reader can expect to be primed with an insightful understanding of the indications, results, and limitations of checkpoint blockade for NSCLC and may recognize and capture an opportunity for innovation to advance this field.

Bryan Burt, $M D$

\section{HISTORY OF TUMOR IMMUNOTHERAPY}

Harnessing the power of the immune system to control cancer is uniquely embedded in surgical history. Having noticed the regression of certain malignancies after the development of local infection William B. Coley, practicing in the late 1800s in what is now Memorial Sloan Kettering Cancer Center, began treating patients with mixtures of live and inactivated Streptococcus pyogenes and Serratia marcescens. ${ }^{2}$ While he reported dramatic results in some (Figure 1), limited reproducible success was achieved in others. Such data, however, spurred the use of the tuberculosis vaccine Bacillus Calmette-Guérin (or BCG) for the treatment of superficial bladder cancer, a practice that is in use by urologic surgeons today. ${ }^{3}$ The discovery that infectious agents control malignancies via activation of the immune system inspired more direct approaches toward immunotherapy. Spurred by the discovery that the growth factor interleukin-2 (IL-2) could result in dramatic expansion of T lymphocytes, the National Cancer Institute Surgery Branch led by Steve Rosenberg spearheaded clinical 

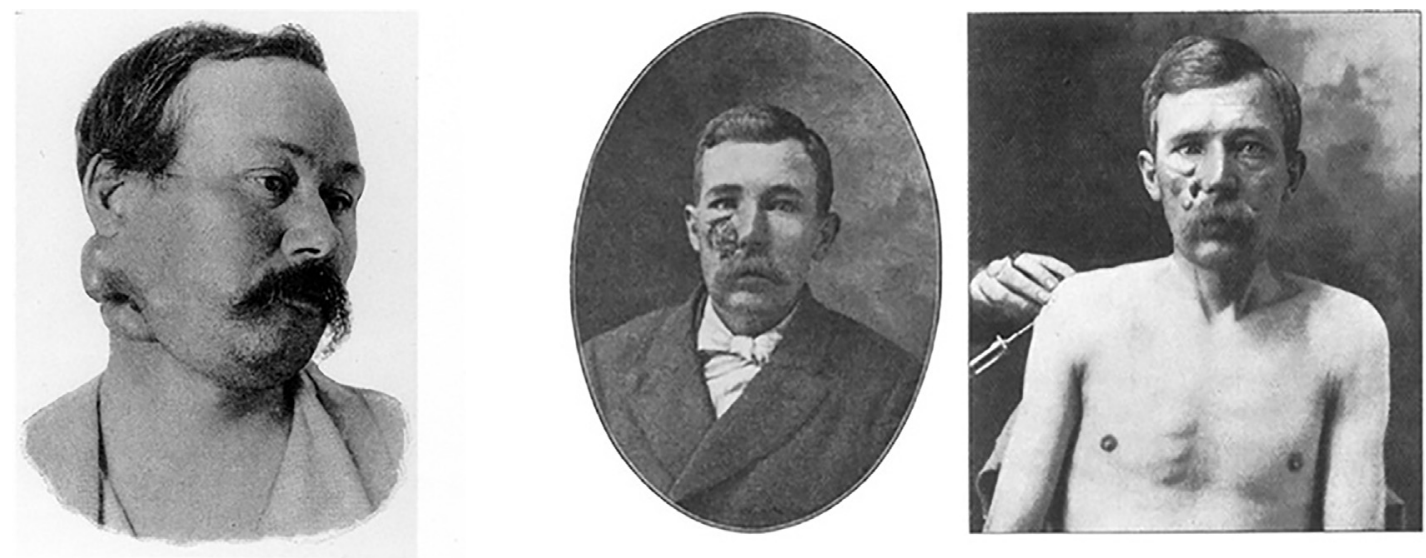

FIGURE 1. An Italian immigrant named Zola was treated successfully with 1 injection of Coley's toxin (left). Other patients needed multiple injections before their tumors would respond (middle and right). Figure adapted from Cancer Research Institute archives/Proceedings of the Royal Society of Medicine 1910.

trials of high-dose IL-2. Administration of this cytokine to those with treatment-resistant metastatic melanoma and renal cell carcinoma led to a near-miraculous long-term remission of disease in $7 \%$ to $9 \%$ of patients. To date, high-dose IL-2 therapy represents the only reproducible source of curative immunotherapy. ${ }^{4}$ The US Food and Drug Administration approved high-dose IL-2 in the 1990s for use in metastatic melanoma and renal cell carcinoma. Nevertheless, despite these exciting results, most immunotherapy fails to eradicate solid tumors.

\section{HISTORY OF CHECKPOINT BLOCKADE IMMUNOTHERAPY}

The tumor microenvironment represents a complex interplay of malignant cells along with nonhematopoietic and myeloid stromal cells as well as immunomodulatory cytokines. The key discovery that paved the way for modern checkpoint blockade immunotherapy was made in the 1990s by Jim Allison's group in UC Berkeley. Along with graduate student Max Krummel and postdoctoral fellow Cynthia Chambers, Allison made the key discovery that after initial activation $\mathrm{T}$ cells express the inhibitory molecule cytotoxic T-lymphocyte-associated protein-4 (CTLA-4), whose main purpose is to prevent exuberant overactivation of lymphocytes. Tumor-associated T lymphocytes are especially enriched for its expression, consistent with their hypoactive state. Although constitutive CTLA-4 knockout mice succumb to massive immunoactivation and death early in life, transient antibody blockade had minimal side effects and improved immunologic control of cancer in small animal models. ${ }^{5}$ This finding led to the birth of the new field of checkpoint-blockade immunotherapy by spurring both the discovery of many new regulatory molecules expressed on the surface of lymphocytes that function as inhibitors of T-cell activation or checkpoint regulators (Figure 2). The translation of the humanized anti-CTLA-4 antibody to the clinic as ipilimumab (Bristol-Myers Squibb, Princeton, NJ) has demonstrated great success in the control of malignant melanoma resistant to other forms of therapy. ${ }^{6}$

\section{CURRENT STATUS OF CHECKPOINT BLOCKADE IMMUNOTHERAPY IN LUNG CANCER}

Limited data from smaller trials have led to speculation that checkpoint blockade immunotherapy might offer a survival advantage to certain patients with lung cancer. The prevalence of the inhibitory receptor programed death ligand-1 (PD-L1) on lung cancer and its receptor programmed cell death protein-1 (PD-1) in lung cancer-

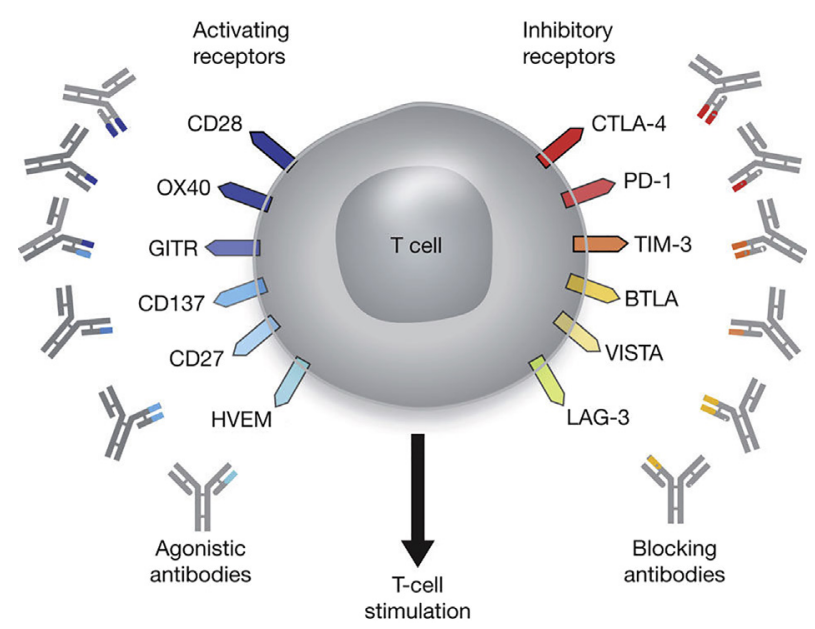

FIGURE 2. It is now accepted that a summation of signals from activating and inhibitory (checkpoint) signals dictate the fate and activation status of the T cell. $C D 28$, Cluster of differentiation 28; $O X 40$, a member of the tumor necrosis factor receptor superfamily-CD134; GITR, glucocorticoid-induced TNFR-related protein; HVEM, herpesvirus entry mediator; $C T L A-4$, cytotoxic T-lymphocyte-associated antigen $4 ; P D$-1, programmed death 1 receptor; TIM-3, T-cell immunoglobulin and mucin-domain containing-3; BTLA, $\mathrm{B}$ and T lymphocyte associated; VISTA, V-domain Ig-containing suppressor of T-cell activation; $L A G-3$, lymphocyte-activation gene 3 . 
infiltrating lymphocytes made this pathway particularly prime for targeting, ${ }^{7}$ specifically since PD-L1/PD-1 interactions downregulate immune responses through multiple mechanisms. ${ }^{8,9}$ Three recent large, prospective randomized clinical trials, KEYNOTE-24 funded by Merck (Kenilworth, NJ), CheckMate 026 by Bristol-Myers Sqibb, and PACIFIC funded by AstraZeneca (Cambridge, United Kingdom), now establish PD-1 checkpoint blockade as a viable therapeutic modality for select patients with lung cancer. ${ }^{10-12}$ Investigators from PACIFIC randomized more than 700 patients who had completed chemoradiation for locally advanced and unresectable lung cancer to receive immunotherapy in the form of durvalumab, a humanized monoclonal antibody that blocks the interaction of PD-L1 with PD-1 (Figure 2). Patients, assigned at a 2:1 ratio, to durvalumab or placebo, demonstrated a significant prolongation of survival or time until metastatic disease (23.2 vs 14.6 months) and had an overall increase in 18 months' progression-free survival $(44.2 \%$ vs $27 \%))^{12}$ In KEYNOTE-24, investigators similarly randomized more than 300 patients with previously untreated stage IV lung cancer to receive either pembrolizumab, a humanized monoclonal antibody against PD-1, or standard-of-care chemotherapy. The overall response rate was greater in the pembrolizumab compared with the chemotherapy group $(47.8 \%$ vs $27.8 \%)$, and the overall survival was longer in the immunotherapy arm. ${ }^{10}$ Investigators from CheckMate 026 enrolled more than 400 patients with previously treated metastatic or recurrent lung cancer to receive nivolumab, a humanized anti-PD-1, or standard-of-care chemotherapy. Unlike KEYNOTE-24, no difference in survival was detected between the immune- and chemotherapy arms in CheckMate 026. ${ }^{11}$

Careful analysis of these 3 trials points out the possible etiology of the discrepancies in outcome. For example, patients in KEYNOTE-24 had PD-L1 expression of 50\% or more on tumor cells, whereas patients with much lower expression levels were enrolled in CheckMate 026. Such discrepancy might act to explain the improvement of immune over chemotherapy in KEYNOTE-24 but not CheckMate 026. Although both forms of the humanized antibodies used in these 2 trials targeted PD-1 expressed on T cells, they were dosed in different regimens and most likely bound different regions of the extracellular PD-1 domain. Thus, the degree of PD-1/PD-L1 blockade may vary between the 2 studies. Nevertheless, these exciting data now definitively demonstrate that checkpoint blockade has a definitive role in the treatment of lung cancer.

\section{FUTURE PROSPECTS FOR IMMUNOTHERAPY IN LUNG CANCER}

In addition to the aforementioned trials, multiple aspects of PD-1 checkpoint blockade are currently being explored.
The use of pembrolizumab, as evaluated in KEYNOTE024, for induction neoadjuvant therapy in patients with resectable lung cancer opened for accrual in Germany in August 2017 (NEOMUN trial). A similar study comparing PD-1 blockade by nivolumab versus concurrent PD-1 and CTLA-4 blockade by combination nivolumab/ipilimumab is open for accrual at MD Anderson Cancer Center. ${ }^{13}$ Despite the current frenzy of excitement of checkpoint blockade as the ultimate, and often sole form of immunotherapy, we must not lose sight of the fact that successful immunologic therapy for cancer will most likely rely on concomitant targeting of multiple overlapping and independent pathways. This has been the case for treatment of HIV, where multiple overlapping antiretroviral drugs target several aspects of the HIV life cycle, ${ }^{14}$ successful immunosuppression for solid organ transplantation, where a calcineurin inhibitors are combined with corticosteroids and an antimetabolite, ${ }^{15}$ as well as curative induction therapy for esophageal cancer, where chemo and radiation therapy are combined with surgical resection. ${ }^{16}$ Thus, a rational approach for combination immunotherapy would most likely combine checkpoint blockade with concomitant immunostimulation by cytokine therapy ${ }^{4}$ or agonistic antibodies. ${ }^{17}$

\section{Conflict of Interest Statement}

Authors have nothing to disclose with regard to commercial support.

\section{References}

1. Cavnar S, Valencia P, Brock J, Wallenstein J, Panier V. The immunooncology race: myths and emerging realities. Nat Rev Drug Discov. 2017; 16:83-4.

2. Coley W. The treatment of malignant tumors by repeated innoculations of erysipelas: with a report of ten original cases. Am J Med Sci. 1893;10:487-511.

3. Morales A, Eidinger D, Bruce AW. Intracavitary Bacillus Calmette-Guerin in the treatment of superficial bladder tumors. J Urol. 1976;116:180-3.

4. Rosenberg SA. IL-2: the first effective immunotherapy for human cancer. J Immunol. 2014;192:5451-8.

5. Leach DR, Krummel MF, Allison JP. Enhancement of antitumor immunity by CTLA-4 blockade. Science. 1996;271:1734-6.

6. Hodi FS, O’Day SJ, McDermott DF, Weber RW, Sosman JA, Haanen JB, et al. Improved survival with ipilimumab in patients with metastatic melanoma. $N$ Engl J Med. 2010;363:711-23.

7. Scheel AH, Ansen S, Schultheis AM, Scheffler M, Fischer RN, Michels S, et al. PD-L1 expression in non-small cell lung cancer: correlations with genetic alterations. Oncoimmunology. 2016;5:e1131379.

8. Sharpe AH, Pauken KE. The diverse functions of the PD1 inhibitory pathway. Nat Rev Immunol. November 13, 2017 [Epub ahead of print].

9. Krupnick AS, Gelman AE, Barchet W, Richardson S, Kreisel FH, Turka LA, et al. Murine vascular endothelium activates and induces the generation of allogeneic CD4+25+Foxp3+ regulatory $\mathrm{T}$ cells. J Immunol. 2005;175: 6265-70.

10. Reck M, Rodriguez-Abreu D, Robinson AG, Hui R, Csőszi T, Fülöp A, et al Pembrolizumab versus chemotherapy for PD-L1-positive non-small-cell lung cancer. N Engl J Med. 2016;375:1823-33.

11. Carbone DP, Reck M, Paz-Ares L, Creelan B, Horn L, Steins M, et al. First-line nivolumab in stage IV or recurrent non-small-cell lung cancer. $N$ Engl J Med. 2017;376:2415-26.

12. Antonia SJ, Villegas A, Daniel D, Vicente D, Murakami S, Hui R, et al. Durvalumab after chemoradiotherapy in stage III non-small-cell lung cancer. $N$ Engl $J$ Med. 2017;377:1919-29. 
13. M.D. Anderson Cancer Center. Phase II study of induction checkpoint blockade for untreated stage I-IIIA non-small cell lung cancers amenable for surgical resection, NEOSTAR/INDUCTION - strategic alliance: BMS. ClinicalTrials. gov Identifier: NCT03158129. Available at: https://clinicaltrials.gov/ct2/show/ NCT03158129?term $=$ ipilimumab\&recrs $=$ ab\&cond $=$ Lung + Cancer $\% 2 C+$ Nonsmall + Cell\&cntry $=$ US \& state $=$ US $\% 3$ ATX \& city $=$ houston $\&$ rank $=1$. Accessed January 15, 2018.

14. Fauci AS, Folkers GK. Toward an AIDS-free generation. JAMA. 2012;308: $343-4$
15. Duncan MD, Wilkes DS. Transplant-related immunosuppression: a review of immunosuppression and pulmonary infections. Proc Am Thorac Soc. 2005;2: 449-55.

16. Walsh TN, Noonan N, Hollywood D, Kelly A, Keeling N, Hennessy TP. A comparison of multimodal therapy and surgery for esophageal adenocarcinoma. $N$ Engl J Med. 1996;335:462-7.

17. Linch SN, McNamara MJ, Redmond WL. OX40 Agonists and combination immunotherapy: putting the pedal to the metal. Front Oncol. 2015; $5: 34$. 